Article

\title{
Low-altitude sensing of urban atmospheric turbulence with UAV
}

\author{
Alexander Shelekhov 1,*, Aleksey Afanasiev ${ }^{2}$, Evgenia Shelekhova ${ }^{1}$, Alexey Kobzev ${ }^{1}$, Alexey Tel'minov ${ }^{1}$, Alex- \\ ander Molchunov ${ }^{1}$ and Olga Poplevina ${ }^{1}$
}

1 Institute of Monitoring of Climatic and Ecological Systems SB RAS, 634055, Tomsk, Russia, 10/3, Academichesky Ave

2 V.E. Zuev Institute of Atmospheric Optics SB RAS, 634055, Tomsk, Russia, 1, Academician Zuev square

* Correspondence: ash@imces.ru; Tel.: +7-952-883-9923

\begin{abstract}
The capabilities of a quadcopter in the hover mode for low-altitude sensing of atmospheric turbulence with high spatial resolution in urban areas characterized by complex orography are investigated. The studies were carried out in different seasons (winter, spring, summer, and fall), and the quadcopter hovered in the immediate vicinity of ultrasonic weather stations. The DJI Phantom 4 Pro quadcopter and AMK-03 ultrasonic weather stations installed in different places of the studied territory were used in the experiment. The smoothing procedure was used to main regularities in the behavior of the longitudinal and lateral spectra of turbulence in the inertial and energy production ranges. The longitudinal and lateral turbulence scales were estimated by the least-square fit method with the von Karman model as a regression curve. It is shown that the turbulence spectra obtained with DJI Phantom 4 Pro and AMK-03 generally coincide with minor differences observed in the high-frequency region of the spectrum. In the inertial range, the behavior of the turbulence spectra shows that they obey the Kolmogorov-Obukhov " $5 / 3$ " law. In the energy production range, the longitudinal and lateral turbulence scales and their ratio measured by DJI Phantom 4 Pro and AMK-03 agree to a good accuracy. Discrepancies in the data obtained with the quadcopter and the ultrasonic weather stations at the territory with complex orography are explained by the partial correlation of the wind velocity series at different measurement points and the influence of the inhomogeneous surface.
\end{abstract}

Keywords: quadcopter; ultrasonic weather station; turbulence, longitudinal and lateral spectra, scales, urban environment

\section{Introduction}

Recently, unmanned aerial vehicles (UAVs) have become widespread in our life, and they are now an important component of the airspace. Analysis and forecast of modern trends in their use show that in the next decade there will be an explosive growth in the number of commercial and military UAVs. A main deterrent to the use of UAVs in the context of a sharp increase in their number is the lack of an efficient system for control of UAV movement under bad weather conditions [1-6]. Atmospheric turbulence is the main factor that most strongly affects the efficiency of future UAVs traffic management systems. Many UAVs are small in size and light in weight. As a result, their trajectory can deviate significantly in a turbulent atmosphere, and the loss of control is highly probable in this case.

The flight trajectories of a significant part of UAVs lie at altitudes up to $500 \mathrm{~m}$, where the formation of the atmospheric boundary layer is considerably affected by the local orography. For example, for flights in an urban environment, i.e. environment with complex orography, atmospheric turbulence is characterized by strong spatial inhomogeneity due to the presence of buildings, park zones, highways, etc. 
The main technologies to obtain information about turbulence profiles of the urban atmosphere are lidar, sodar and radar sensing methods. For these sensing technologies, the spatial resolution ranges from few tens to hundreds of meters [7-15]. Spatial variations of turbulent air flows can reach several meters, which is much smaller than the spatial resolution of lidars, sodars and radars. This discrepancy between the spatial resolution of the applied technologies and the scale of turbulent flows in the atmosphere can lead to their significant averaging and, consequently, to significant errors in atmospheric turbulence measurements. Thus, the future UAVs traffic management systems must use data on the turbulent atmosphere obtained with high spatial resolution.

Acoustic anemometry methods can be used to obtain data on the atmosphere with high spatial resolution $[11,16,17]$. Complete information about the state of the atmosphere at different heights can be obtained with acoustic anemometry methods if acoustic devices are set on weather towers or a tethered balloon, which is not always possible in an urban environment.

One of the main trends in low-altitude sensing, i.e. to heights of about $500 \mathrm{~m}$, is the development of methods for diagnostics of the turbulent atmosphere with UAVs. The results of diagnostics of the speed of air mass flows with UAV are reported in [18-35]. In [36-46], the fundamental possibility of measuring the turbulence spectra with fixed-wing UAVs of various sizes and weights was shown. In the process of sensing, UAVs of this type move in space for a long time to obtain a representative series of observations of wind velocity fluctuations. This sensing scheme results in the same spatial uncertainty in measurements of turbulence of the urban atmosphere as in the case of lidars, sodars and radars. This uncertainty can be eliminated by using a rotary-wing UAV capable of hovering at a fixed spatial point for a long time.

The results of studying the turbulence spectrum in the inertial and energy production ranges with the DJI Mavic Mini quadcopter in the altitude hold mode are reported in [47]. The measurements were carried out over the territory characterized by the flat and uniform surface with a slight slope; it bordered on a cottage village on one side and a forest on the other side. The results obtained are in a good agreement with the theory of homogeneous and isotropic turbulence and the data measured by the methods of acoustic anemometry.

Knowledge of the state of atmospheric turbulence allows us to study not only its effect on the efficiency of UAV management systems. It is well-known that turbulence reduces the possibility of efficient use of wind energy and causes accelerated wear. Techniques of low-altitude sensing of the atmospheric state, including turbulence, allow us to estimate the climate change caused by urban growth and are needed to address current and future urbanization challenges.

In this paper, we study the capabilities of a quadcopter for monitoring the state of atmospheric turbulence in an urban area over the territory with complex orography. One of the main fundamental parameters, which describes the state of atmospheric turbulence quite completely and accurately and which is investigated in this paper, is the turbulence spectrum [48-52]. The theoretical part of the paper describes the coordinate systems, introduces the concept of a spectral tensor of turbulence, and presents the Taylor hypothesis, which allows us to obtain a relationship between the spatial and temporal spectra of turbulence, and the basic equations of the von Karman model. In this part, we derive the equations for the wind velocity components measured with the quadcopter in the hover mode, as well as the equations for longitudinal and lateral velocity fluctuations.

The second part of the paper provides general information about the experiment, presents the results of measurements of the quadcopter speed and the longitudinal and lateral components of the wind velocity measured with the DJI Phantom 4 Pro quadcopter and AMK-03 ultrasonic weather stations [16, 17]. In addition, longitudinal and lateral turbulence spectra measured with the DJI Phantom 4 Pro and AMK-03 are present- 
ed, and their behavior in the inertial and energy production ranges is studied. In Conclusion, the main results are summarized.

\section{Coordinate systems}

Figure 1 shows schematically the arrangement of the equipment and the direction of the average wind speed during the experiments. It can be seen that the AMK-03 weather station and the quadcopter are oriented differently relative to each other and relative to the direction of the average wind. The ultrasonic weather station is oriented in space along the cardinal points, and the quadcopter can be oriented arbitrarily. Thus, in the case of AMK-03, the measured wind speed data correspond to the $\{\mathrm{E}, \mathrm{N}\}$ coordinate system used in meteorology, in which one axis is directed to the east (E) and the other axis is directed to the north (N) [49].

\section{DJI Phantom 4 Pro}

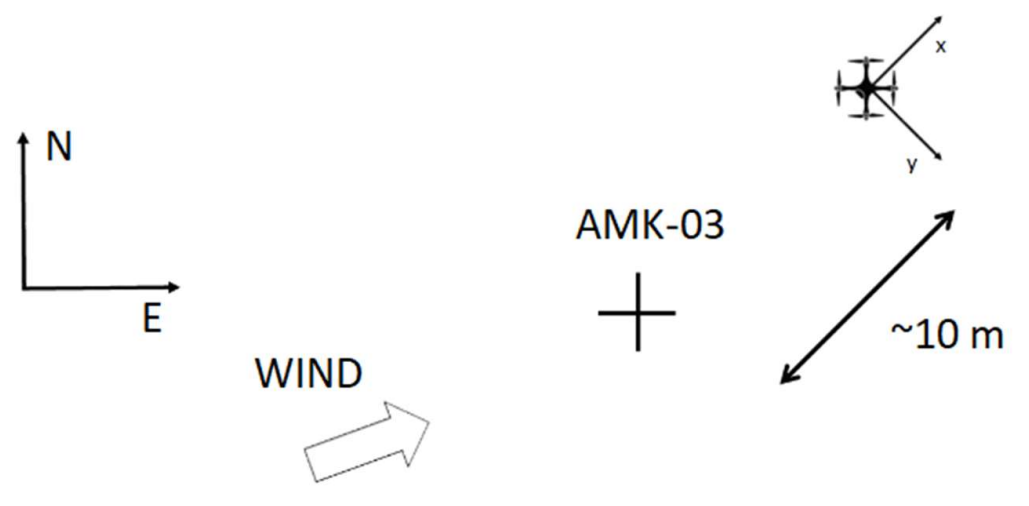

Figure 1. Block diagram of the experiments.

The quadcopter dynamics may be described in a coordinate system other than the $\{$ E, N\} meteorological system. Denote the coordinate system, in which the quadcopter dynamics is described, as $\{x, y\}$. The axes of this coordinate system are shown as $x$ and $y$ in Fig. $1[21,31]$.

In the theory of turbulence [48-51], fluctuations of the wind velocity are described in the coordinate system related to the mean wind. In the coordinates system related to the mean horizontal wind, one axis is directed along the mean wind, and the two other coordinate axes are directed normally to the mean wind. As a result of this choice of the coordinate system, longitudinal and two lateral fluctuations of the wind velocity are clearly distinguished.

\section{Taylor hypothesis}

It is well known that in the atmosphere the horizontal air mass transfer often prevails over the vertical motion. The average vertical component of the wind speed is small and can be neglected. Thus, we can take into account only the turbulent vertical component. In this case, one of the axes of the coordinate system can be directed along the mean horizontal wind. Two other axes are directed normally to the mean horizontal wind, and one of them lies in a horizontal plane, while another is directed vertically upward. As a result, in this coordinate system related to the mean horizontal wind, the velocity field of the turbulent air flow at the point $\mathbf{r}=\{\xi, 0,0\}$ has the form [48-51]:

$$
\mathrm{u}(\mathbf{r}, t)=\langle\mathrm{u}\rangle+\mathrm{u}^{\prime}(\mathbf{r}, t),
$$




$$
\begin{gathered}
\mathrm{v}(\mathbf{r}, t)=\mathrm{v}^{\prime}(\mathbf{r}, t), \\
\mathrm{w}(\mathbf{r}, t)=\mathrm{w}^{\prime}(\mathbf{r}, t),
\end{gathered}
$$

where $\langle\mathrm{u}\rangle$ is the mean wind speed, $\mathrm{u}^{\prime}(\mathbf{r}, t), \mathrm{v}^{\prime}(\mathbf{r}, t)$, and $\mathrm{w}^{\prime}(\mathbf{r}, t)$ are fluctuations of the wind speed, $\langle\ldots\rangle$ is the operator of statistical averaging. It can be seen from Eqs. (1)-(3) that longitudinal fluctuations of the wind speed $\mathrm{u}^{\prime}(\mathbf{r}, t)$ are directed along the direction of the mean wind speed, and two lateral fluctuations of the wind speed $\mathrm{v}^{\prime}(\mathbf{r}, t)$ and $\mathrm{w}^{\prime}(\mathbf{r}, t)$ are directed normally to the mean wind speed. The component $\mathrm{v}^{\prime}(\mathbf{r}, t)$ lies in the horizontal plane, and the component $w^{\prime}(\mathbf{r}, t)$ is directed vertically upward and describes vertical fluctuations.

In the mathematical description of fluctuations of the velocity field, the concepts of the second-rank correlation tensor and the turbulence spectrum tensor are introduced [48-51]. For isotropic turbulence, the diagonal elements of the correlation tensor can be written in the form

$$
\begin{aligned}
& B_{\mathrm{u}}(\xi ; t)=\left\langle\mathrm{u}^{\prime}(\xi, 0,0 ; t) \mathrm{u}^{\prime}(0,0,0 ; 0)\right\rangle, \\
& B_{\mathrm{v}}(\xi ; t)=\left\langle\mathrm{v}^{\prime}(\xi, 0,0 ; t) \mathrm{v}^{\prime}(0,0,0 ; 0)\right\rangle, \\
& B_{\mathrm{w}}(\xi ; t)=\left\langle\mathrm{w}^{\prime}(\xi, 0,0 ; t) \mathrm{w}^{\prime}(0,0,0 ; 0)\right\rangle .
\end{aligned}
$$

The diagonal components of the one-dimensional spatial spectral tensor of turbulence take the form

$$
\begin{aligned}
& \phi_{\mathrm{u}}(\kappa)=\int_{-\infty}^{\infty} B_{\mathrm{u}}(\xi ; t) e^{2 \pi i \kappa \xi} d \xi, \\
& \phi_{\mathrm{v}}(\kappa)=\int_{-\infty}^{\infty} B_{\mathrm{v}}(\xi ; t) e^{2 \pi i \kappa \xi} d \xi, \\
& \phi_{\mathrm{w}}(\kappa)=\int_{-\infty}^{\infty} B_{\mathrm{w}}(\xi ; t) e^{2 \pi i \kappa \xi} d \xi,
\end{aligned}
$$

and the diagonal components of the temporal spectral tensor of turbulence are determined by the equations

$$
\begin{aligned}
& \Phi_{\mathrm{u}}(f)=\int_{-\infty}^{\infty} B_{\mathrm{u}}(\xi ; t) e^{2 \pi i f t} d t \\
& \Phi_{\mathrm{v}}(f)=\int_{-\infty}^{\infty} B_{\mathrm{v}}(\xi ; t) e^{2 \pi i} d t \\
& \Phi_{\mathrm{w}}(f)=\int_{-\infty}^{\infty} B_{\mathrm{w}}(\xi ; t) e^{2 \pi i f t} d t
\end{aligned}
$$

The diagonal components of the temporal spectral tensor of turbulence $\Phi_{\mathrm{u}}(f)$ and $\Phi_{\mathrm{v}}(f)$ are the longitudinal and lateral spectra of turbulence, and those of $\Phi_{\mathrm{w}}(f)$ form the vertical spectrum of turbulence.

In the coordinate system related to the mean wind, we have the opportunity to use Taylor's hypothesis of "frozen" turbulent fluctuations [48-51]. The essence of this hypothesis is that the entire spatial turbulent pattern moves in time with the mean wind speed $\langle u\rangle$. The application of Taylor's hypothesis leads to the relation between the spa- 
tiotemporal and purely spatial characteristics of fluctuations of the wind velocity field in the form

$$
\begin{aligned}
\mathrm{u}^{\prime}(\xi, 0,0 ; t) & =\mathrm{u}^{\prime}(\xi-\langle\mathrm{u}\rangle t, 0,0), \\
\mathrm{v}^{\prime}(\xi, 0,0 ; t) & =\mathrm{v}^{\prime}(\xi-\langle\mathrm{u}\rangle t, 0,0), \\
\mathrm{w}^{\prime}(\xi, 0,0 ; t) & =\mathrm{w}^{\prime}(\xi-\langle\mathrm{u}\rangle t, 0,0) .
\end{aligned}
$$

The application of Taylor's hypothesis (13)-(15) to Eqs. (7)-(9) with allowance for Eqs. (10)-(12) leads to the well-known relation between the spatial and temporal spectra

$$
\begin{gathered}
\phi_{\mathrm{u}}(\kappa)=\langle u\rangle \Phi_{\mathrm{u}}(f), \\
\phi_{\mathrm{v}}(\kappa)=\langle u\rangle \Phi_{\mathrm{v}}(f), \\
\phi_{\mathrm{w}}(\kappa)=\langle u\rangle \Phi_{w}(f) .
\end{gathered}
$$

The relation between the spatial and temporal frequencies is given as $\kappa=f /\langle u\rangle$. In experiments, we measure temporal spectra, while the theory deals with spatial spectra. Thus, Eqs. (16)-(18) allow us to compare the behavior of experimentally measured temporal spectra with theoretical results.

\section{Model of atmospheric turbulence}

One of the most commonly used turbulence spectra models is the von Karman model [48-52], which allows us to study the behavior of the spectrum in the energy production and inertial ranges. In addition to the von Karman model, a suitable approximation in problems of UAV dynamics in a turbulent atmosphere is the Dryden turbulence model [6]. Other models, such as the unified turbulence model, can also be used to describe atmospheric turbulence [52].

In this study, we use the von Karman model to analyze turbulence spectra. With allowance for Eqs. (16)-(18), the equations for the longitudinal, lateral, and vertical temporal spectra of turbulence for the von Karman model have the form

$$
\begin{gathered}
\frac{\Phi_{\mathrm{u}}(f)}{\sigma_{\mathrm{u}}^{2}}=\frac{2 \mathrm{~L}_{\mathrm{u}}}{\pi} \frac{1}{\left[1+\left(1.339 \mathrm{~L}_{\mathrm{u}} \cdot 2 \pi f /\langle\mathrm{u}\rangle\right)^{2}\right]^{5 / 6}}, \\
\frac{\Phi_{\mathrm{v}}(f)}{\sigma_{\mathrm{v}}^{2}}=\frac{2 \mathrm{~L}_{\mathrm{v}}}{\pi} \frac{1+8 / 3\left(2.678 \mathrm{~L}_{\mathrm{v}} \cdot 2 \pi f /\langle w\rangle\right)^{2}}{\left[1+\left(2.678 \mathrm{~L}_{\mathrm{v}} \cdot 2 \pi f /\langle\mathrm{u}\rangle\right)^{2}\right]^{11 / 6}}, \\
\frac{\Phi_{\mathrm{w}}(f)}{\sigma_{\mathrm{w}}^{2}}=\frac{2 \mathrm{~L}_{\mathrm{w}}}{\pi} \frac{1+8 / 3\left(2.678 \mathrm{~L}_{\mathrm{w}} \cdot 2 \pi f /\langle w\rangle\right)^{2}}{\left[1+\left(2.678 \mathrm{~L}_{\mathrm{w}} \cdot 2 \pi f /\langle\mathrm{u}\rangle\right)^{2}\right]^{11 / 6}},
\end{gathered}
$$

where $L_{u}$ is the longitudinal turbulence scale, $L_{v}$ is the lateral turbulence scale, and $L_{w}$ is the vertical turbulence scale, $\sigma_{\mathrm{u}}^{2}, \sigma_{\mathrm{v}}^{2}$, and $\sigma_{\mathrm{v}}^{2}$ are turbulence intensities.

\section{Wind velocity components}

The dynamic equations for the quadcopter center of gravity can be written in the inertial coordinates associated with the Earth as [21, 31, 53]

$$
\begin{gathered}
\ddot{x}=\left(s_{\phi} s_{\psi}+c_{\phi} s_{\theta} c_{\psi}\right) \frac{T}{m}+\frac{F_{x}}{m} \\
\ddot{y}=\left(-s_{\phi} c_{\psi}+c_{\phi} s_{\theta} s_{\psi}\right) \frac{T}{m}+\frac{F_{y}}{m} \\
\ddot{z}=c_{\phi} c_{\theta} \frac{T}{m}-g+\frac{F_{z}}{m}
\end{gathered}
$$


where $s_{(\bullet)}=\sin (\bullet), c_{(\bullet)}=\cos (\bullet), \phi$ is the roll angle, $\theta$ is the pitch angle, $\psi-$ is the yaw angle, $T$ is the aerodynamic force generated by propellers, $F_{x}, F_{y}$, and $F_{z}$ are the drag force components along the $x, y$, and $z$ axes; $m$ is the quadcopter mass, $g$ is the gravity acceleration.

The components of the drag force along the $\mathrm{x}, \mathrm{y}$, and $\mathrm{z}$ axes, which arises during the quadcopter flight, have the form $[18,21,31,53]$

$$
F_{j}=-c_{j}\left(v_{j}-w_{j}\right)
$$

In the linear case

$$
F_{j}=-\frac{1}{2} \rho C_{j} A_{j} \operatorname{sgn}\left(v_{j}-w_{j}\right) \times\left(v_{j}-w_{j}\right)^{2}
$$

in the square-law case. In Eqs. (25) and (26), $c_{j}$ and $C_{j}$ are the drag coefficient along the $\mathrm{x}, \mathrm{y}$, and $\mathrm{z}$ axes; $j$ is the subscript for enumeration of the orthogonal components of vectors, i.e., $j \in\{x, y, z\}, v_{j}$ are the quadcopter speed components, and $w_{j}$ are components of the turbulent flow velocity in the atmosphere in the coordinate system $\{x, y\}, \rho$ is the air density, $A_{j}$ are the projections of the quadcopter area on the corresponding axe, sgn $(\bullet)$ is the sign function.

Consider the case of ideal hover, which can be achieved by compensating all the forces acting on the quadcopter and at $v_{j}=0$. Equations (22)-(24) can be transformed to the case of ideal hover through their linearization. The roll, pitch, and yaw angles in a turbulent atmosphere are sums of the average and fluctuation components: $\phi=\langle\phi\rangle+$ $\phi^{\prime}, \theta=\langle\theta\rangle+\theta^{\prime}$ и $\psi=\langle\psi\rangle+\psi^{\prime}$. In the small-angle approximation, $\phi, \theta \ll \pi$ and at $\psi^{\prime} \ll$ $\pi$, as well as if the conditions $\ddot{x}=\ddot{y}=\ddot{z}=0$ and $v_{j}=0$ are fulfilled, the equations for estimation of the horizontal velocity components of the wind velocity field $w_{x}$ and $w_{y}$ take the form

$$
\begin{gathered}
w_{x}=-\frac{m g}{c_{x}}\left(\langle\varphi\rangle s_{\langle\psi\rangle}+\langle\theta\rangle c_{\langle\psi\rangle}\right)-\frac{m g}{c_{x}}\left(\varphi^{\prime} s_{\langle\psi\rangle}+\theta^{\prime} c_{\langle\psi\rangle}\right), \\
w_{y}=-\frac{m g}{c_{y}}\left(-\langle\varphi\rangle c_{\langle\psi\rangle}+\langle\theta\rangle s_{\langle\psi\rangle}\right)-\frac{m g}{c_{y}}\left(-\varphi^{\prime} c_{\langle\psi\rangle}+\theta^{\prime} s_{\langle\psi\rangle}\right)
\end{gathered}
$$

in the linear case

$$
\begin{aligned}
& w_{x}=-\operatorname{sgn}\left(\langle\varphi\rangle s_{\langle\psi\rangle}+\langle\theta\rangle c_{\langle\psi\rangle}\right) \sqrt{\frac{2 m g}{\rho C_{x} A_{x}}\left|\left(\langle\varphi\rangle s_{\langle\psi\rangle}+\langle\theta\rangle c_{\langle\psi\rangle}\right)\right|}\left\{1+\frac{\varphi^{\prime} s_{\langle\psi\rangle}+\theta^{\prime} c_{\langle\psi\rangle}}{2\left(\langle\varphi\rangle s_{\langle\psi\rangle}+\langle\theta\rangle c_{\langle\psi\rangle}\right)}\right\}, \\
& w_{y}=-\operatorname{sgn}\left(-\langle\varphi\rangle c_{\langle\psi\rangle}+\langle\theta\rangle s_{\langle\psi\rangle}\right) \sqrt{\frac{2 m g}{\rho C_{x} A_{x}}\left|\left(-\langle\varphi\rangle c_{\langle\psi\rangle}+\langle\theta\rangle s_{\langle\psi\rangle}\right)\right|}\left\{1+\frac{-\varphi^{\prime} c_{\langle\psi\rangle}+\theta^{\prime} s_{\langle\psi\rangle}}{2\left(-\langle\varphi\rangle c_{\langle\psi\rangle}+\langle\theta\rangle s_{\langle\psi\rangle}\right)}\right\}
\end{aligned}
$$

in the square-law case .

It follows from Eqs. (27)-(30) that, regardless of the model of the drag force, the estimates of the horizontal components of the turbulent flow velocity are the sum of the regular and fluctuation parts. The regular part of the estimates is determined by the average values of the roll, pitch and yaw angles, whereas the fluctuation part is proportional to the fluctuations of the roll $\varphi^{\prime}$ and pitch $\theta^{\prime}$ angles.

\section{Longitudinal and lateral velocity fluctuations}

In the case of predominance of the horizontal air mass transfer over the vertical motion, the longitudinal and lateral turbulent fluctuations of the wind velocity take the form

$$
\mathrm{u}^{\prime}=n_{E} w_{E}^{\prime}+n_{N} w_{N}^{\prime}
$$




$$
\begin{gathered}
\mathrm{v}^{\prime}=-n_{N} w_{E}^{\prime}+n_{E} w_{N}^{\prime} \\
\mathbf{n}=\left\{n_{E}, n_{N}, 0\right\}=\left\{\frac{\left\langle w_{E}\right\rangle}{\langle\mathrm{u}\rangle}, \frac{\left\langle w_{N}\right\rangle}{\langle\mathrm{u}\rangle}, 0\right\}
\end{gathered}
$$

for the ultrasonic weather station and

$$
\begin{gathered}
\mathrm{u}^{\prime}=n_{x} w_{x}^{\prime}+n_{y} w_{y}^{\prime} \\
\mathrm{v}^{\prime}=-n_{x} w_{y}^{\prime}+n_{y} w_{x}^{\prime} \\
\mathbf{n}=\left\{n_{x}, n_{y}, 0\right\}=\left\{\frac{\left\langle w_{x}\right\rangle}{\langle\mathrm{u}\rangle}, \frac{\left\langle w_{y}\right\rangle}{\langle\mathrm{u}\rangle}, 0\right\}
\end{gathered}
$$

for the quadcopter. Here, $w_{E}^{\prime}$ and $w_{N}^{\prime}$, are fluctuations of wind velocity components along the $\mathrm{E}$ and $\mathrm{N}$ axes, i.e., data of the ultrasonic weather station; $w_{x}^{\prime}$ and $w_{y}^{\prime}$, are fluctuations of the wind velocity components obtained from the results of quadcopter telemetry; $\left\langle w_{E}\right\rangle$ and $\left\langle w_{N}\right\rangle$ are the average components of the horizontal velocity along the $\mathrm{E}$ and $\mathrm{N}$ axes; $\left\langle w_{x}\right\rangle$ and $\left\langle w_{y}\right\rangle$ are estimates of the velocity components along the $x$ and $y$ axes. Thus, Eqs.(31)-(36) allow us to compare the longitudinal and lateral turbulence spectra measured by the quadcopter and the ultrasonic weather station.

\section{General information about the experiment}

Experimental studies were carried out at the territory of the Institute for Monitoring of Climatic and Ecological Systems of the Siberian Branch of the Russian Academy of Sciences (IMCES SB RAS), which is located in Academgorodok, one of the districts of the city of Tomsk (Russian Federation). This area is a territory with complex orography: it is a forested area with the buildings of Academgorodok institutes and highways. Figure 2 shows a Google map of the experimental area. The arrows show the location of the used AMK-03 ultrasonic weather stations, and the measurement dates are indicated.

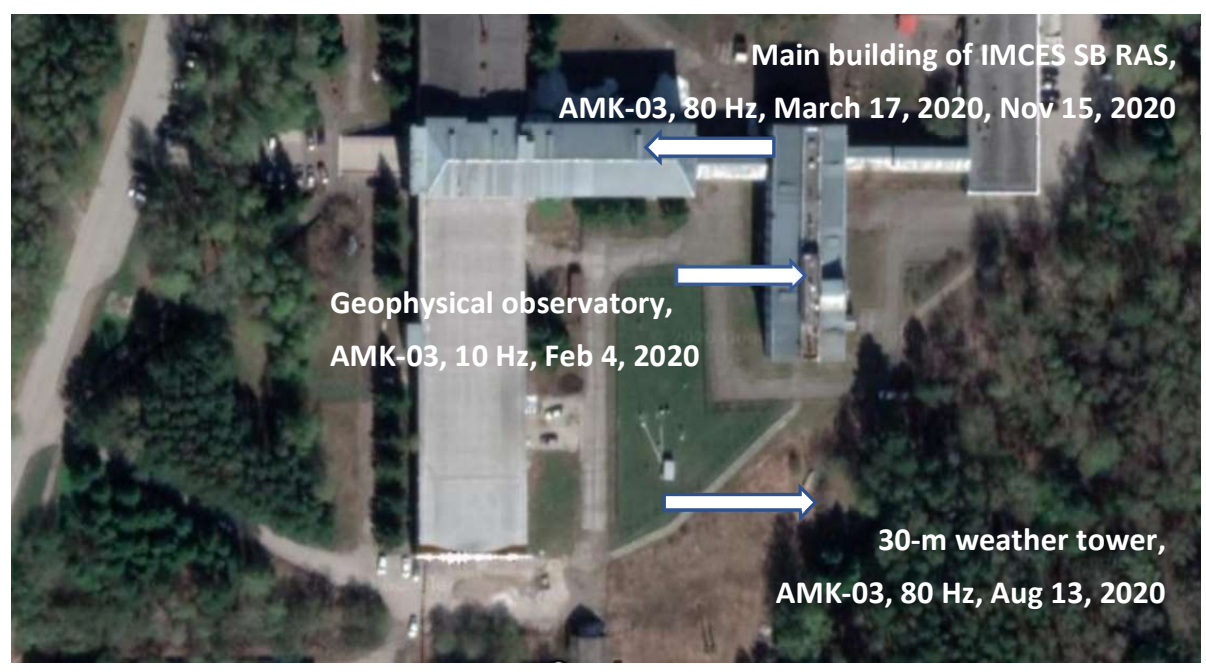

Figure 2. Arrangement of ultrasonic weather stations.

The studies were carried out in different seasons: winter (February 4, 2020), spring (March 17, 2020), summer (August 13, 2020), and fall (November 15, 2020). The quadcopter hovered at different places of the territory with complex orography: over the building of the Geophysical Observatory and the IMCES SB RAS main building, as well as in the immediate vicinity of a small grove where a $30-\mathrm{m}$ weather tower is installed (see Fig. 3). In the experiment on February 20, the launch point of the DJI Phantom 4 Pro 
quadcopter was chosen in close proximity to the foundation of the Geophysical Observatory building, and on August 13 the quadcopter started from the foundation of the 30$\mathrm{m}$ weather tower. During the measurements on March 17 and November 15, the quadcopter took off in the immediate vicinity of the foundation of the IMCES SB RAS main building.

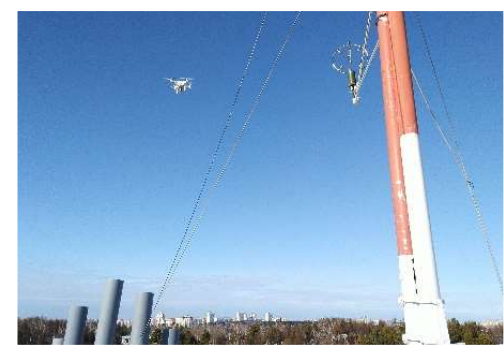

(a)

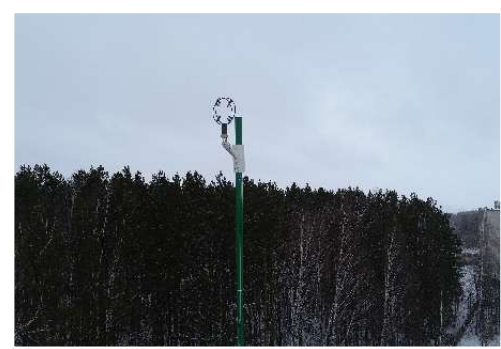

(b)

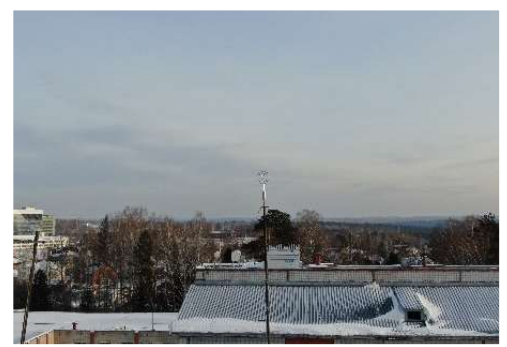

(c)

Figure 3. Quadcopter hovering over the building of the Geophysical Observatory (a); photos of the ultrasonic weather stations installed on the 30-m weather tower (b) and on the IMCES SB RAS main building (c).

The AMK-03 ultrasonic weather station serves to measure and record the wind speed and direction by acoustic anemometry methods, as well as temperature, relative air humidity, and atmospheric pressure [16, 17]. We used AMK-03 of two types, which recorded the wind speed and direction with a frequency of $10 \mathrm{~Hz}$ and $80 \mathrm{~Hz}$. The locations of the weather stations of different types are shown by the arrows in Fig. 2. The data on the state of the quadcopter in the flight logs were recorded at a frequency of 10 $\mathrm{Hz}$.

Figure 4 shows the quadcopter flight paths during the experiments. Table 1 presents the dates and times of the start and end of the experiments, as well as the DJI Phantom 4 Pro quadcopter flight heights. After takeoff, the quadcopters flew up to the AMK-03 ultrasonic weather stations located on the roofs of the buildings and on the weather tower. After the end of the experiments, the quadcopters returned to the starting point.

According to the data of the Tomsk International Airport spaced by $\sim 10 \mathrm{~km}$ from IMCES SB RAS, the following weather conditions were observed during the experiments. On February 4, weather conditions were satisfactory in terms of the quadcopter flight: south-south-west wind, speed of $3 \mathrm{~m} / \mathrm{s}$, air temperature of $-4^{\circ} \mathrm{C}$, air humidity of $86 \%$, light snow, horizontal visibility range of $8 \mathrm{~km}$. On March 17, good weather conditions were recorded: variable wind, speed of $1 \mathrm{~m} / \mathrm{s}$, air temperature of $-1^{\circ} \mathrm{C}$, air humidity of $55 \%$, no precipitation, horizontal visibility range of $10 \mathrm{~km}$ or more. On August 13, the weather in the airport was excellent: southeast wind, speed of $4 \mathrm{~m} / \mathrm{s}$, air temperature of $27^{\circ} \mathrm{C}$, air humidity of $42 \%$, no precipitation, horizontal visibility range of $10 \mathrm{~km}$ or more. On November 15, the weather in the airport was satisfactory in terms of the quadcopter flight: south-south-west wind, speed of $2 \mathrm{~m} / \mathrm{s}$, air temperature of $-9^{\circ} \mathrm{C}$, air humidity of $90 \%$, no precipitation, horizontal visibility range of $10 \mathrm{~km}$ or more. 


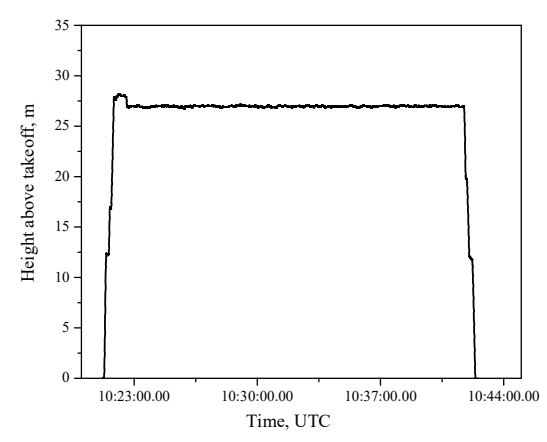

(a)

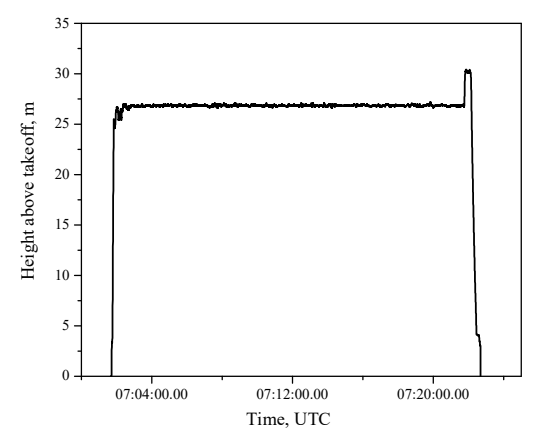

(c)

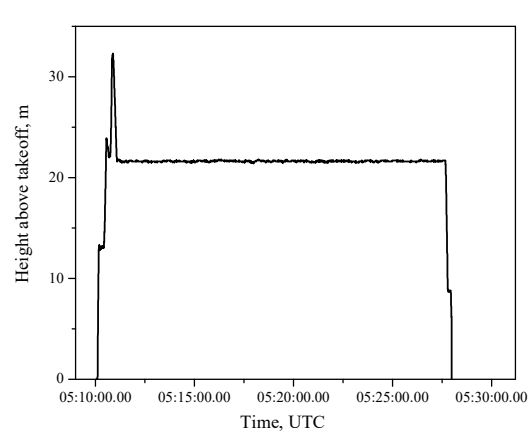

(b)

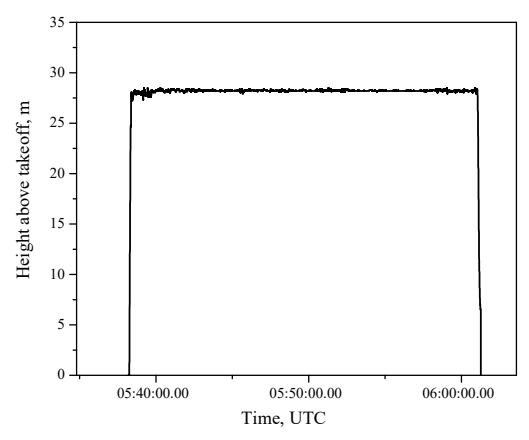

(d)

Figure 4. Quadcopter flight trajectory during the experiments on (a) February 4, (b) March 17, (c) August 13, and (d) November, 15, 2020

Table 1. Date and time of the start and end of the study and the hover height.

\begin{tabular}{cccc}
\hline Date & Start, UTC & End, UTC & Hover height, m \\
\hline Feb 4, 2020 & $10: 21$ & $10: 42$ & 27 \\
Mar 17, 2020 & $05: 10$ & $05: 28$ & 22 \\
Aug 13, 2020 & $07: 01$ & $07: 23$ & 27 \\
Nov 15, 2020 & $05: 38$ & $06: 01$ & 28 \\
\hline
\end{tabular}

Thus, the experiments were carried out in different seasons, under different weather conditions, and the hover took place at different places of the IMCES SB RAS territory, which is characterized by complex orography.

\section{Quadcopter velocity}

Figure 5 shows the dynamics of the $v_{x}, v_{y}$, and $v_{z}$ components of the quadcopter velocity during hovering. It can be seen that, generally, the quadcopter velocity components are equal to zero during the measurements. In short periods of time, the forces acting on the quadcopter exceed the capabilities of the control system and high-precision positioning is disrupted. After regaining the control, the quadcopter begins to move to its original position and, upon reaching this position, it stops. 


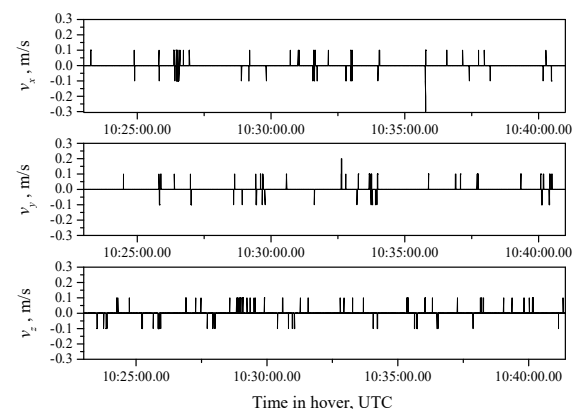

(a)

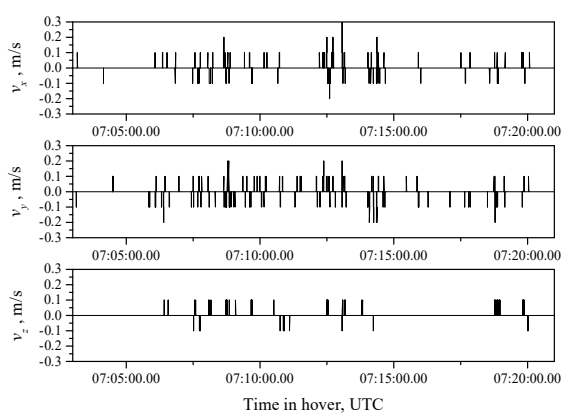

(c)
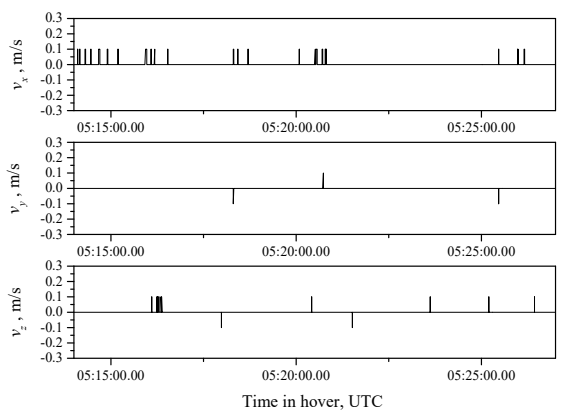

(b)
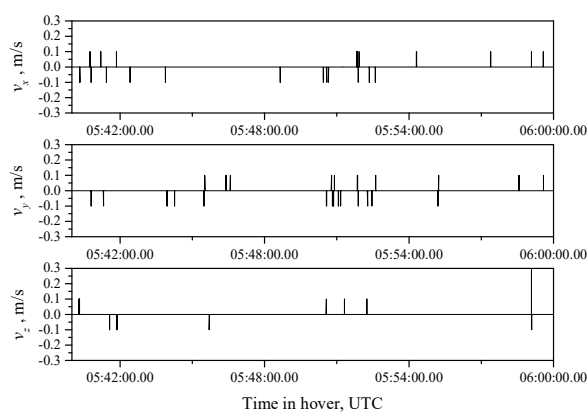

(d)

Figure 5. Quadcopter velocity components along the $x, y$, and $z$ axes during hovering. (a) February 4, (b) March 17, (c) August 13, and (d) November, 15, 2020.

Thus, the periods, in which the precision positioning of the quadcopter in space is disrupted, can be neglected due to their insignificance, and we can believe that ideal hovering was observed during the experiment.

\section{Longitudinal and lateral wind velocity components}

Consider the behavior of the estimates of the longitudinal and lateral components of the wind velocity from the quadcopter data in the altitude hold mode in the turbulent atmosphere and compare it with the results obtained from the data of the AMK-03 ultrasonic weather station.

Figure 6 shows the temporal dynamics of the longitudinal and lateral wind velocity components measured with AMK-03 (red curves) and DJI Phantom 4 Pro (black curves) on (a) February 4, (b) March 17, (c) August 13, and (d) November, 15, 2020. It follows from Fig. 6 that the time series of $u$ and $v$ measured in different ways generally coincide, and discrepancies are observed only in the high-frequency range of fluctuations. 

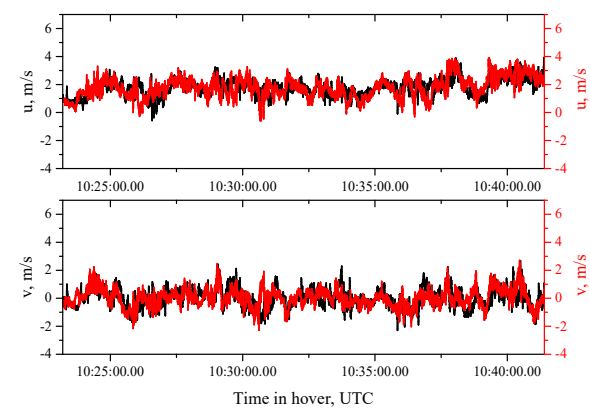

(a)

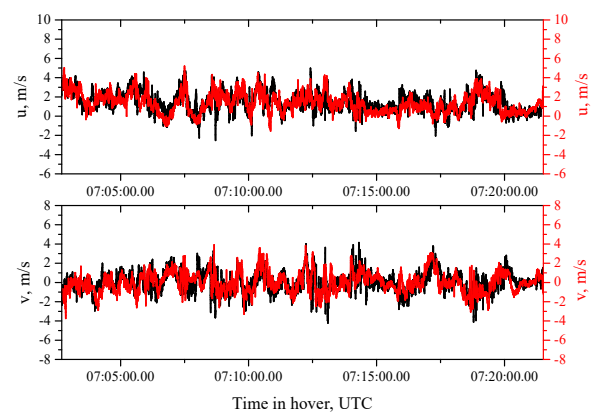

(c)
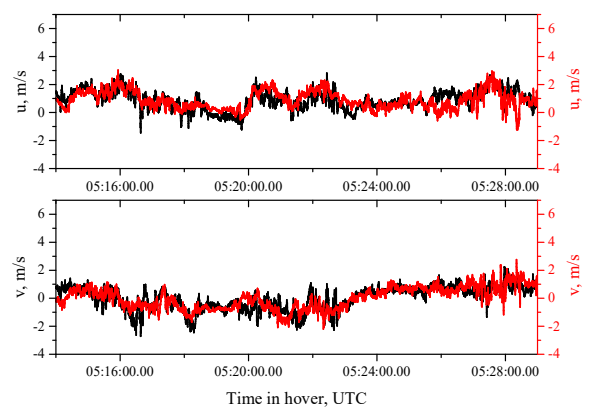

(b)

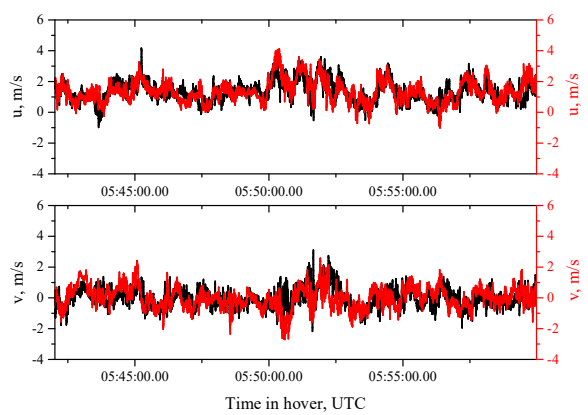

(d)

Figure 6. Temporal dynamics of the longitudinal and lateral components of wind velocity: quadcopter data (black curve) and AMK-03 data (red curve) on (a) February 4, (b) March 17, (c) August 13, and (d) November, 15, 2020.

Table 2 presents the average values of the longitudinal, lateral and vertical components of the wind velocity measured with AMK-03 in the experiments. It can be seen that the average wind speed $\langle\mathrm{u}\rangle$ differs from the corresponding values observed at the airport. This difference is explained by two circumstances. First, the airport is located approximately $10 \mathrm{~km}$ far from the IMCES SB RAS buildings. Second, the airport territory, where the measurements were carried out, has a flat underlying surface, whereas the IMCES SB RAS territory has complex orography.

It can also be seen from Table 2 that in the experiment, the horizontal transfer of air masses predominated over the vertical motion, i.e. $\langle w\rangle \approx 0$. The fulfillment of the condition $\langle w\rangle \approx 0$ in the experiment means that the assumption of the predominance of the horizontal air mass transfer over the vertical motion when calculating longitudinal and lateral turbulent fluctuations of the wind velocity by Eqs. (31)-(36) is justified. It should be noted that the maximal values of the longitudinal and lateral components of the wind velocity exceed the average wind, which is indicative of strong turbulence during measurements.

Table 2. Average values of the longitudinal, lateral, and vertical wind velocity components, and their maximal values

\begin{tabular}{cccccc}
\hline Date & $\langle\mathbf{u}\rangle, \mathbf{m} / \mathbf{s}$ & $\langle\mathbf{v}\rangle, \mathbf{m} / \mathbf{s}$ & $\mathbf{m a x}(\mathbf{u}), \mathbf{m} / \mathbf{s}$ & $\mathbf{m a x}(\mathbf{v}), \mathbf{m} / \mathbf{s}$ & $\langle\mathbf{w}\rangle, \mathbf{m} / \mathbf{s}$ \\
\hline Feb 4, 2020 & 1.78 & 0 & 4.9 & 2.6 & 0.3 \\
Mar 17, 2020 & 0.89 & 0 & 3.03 & 2.76 & 0.04 \\
Aug 13, 2020 & 1.36 & 0 & 5.20 & 3.91 & -0.1 \\
Nov 15, 2020 & 1.35 & 0 & 4.13 & 2.58 & 0.15 \\
\hline
\end{tabular}




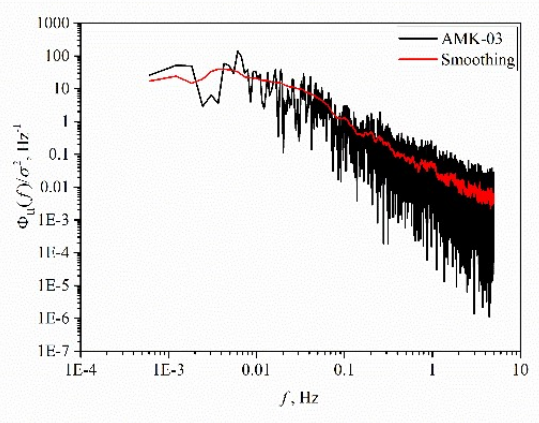

(a)

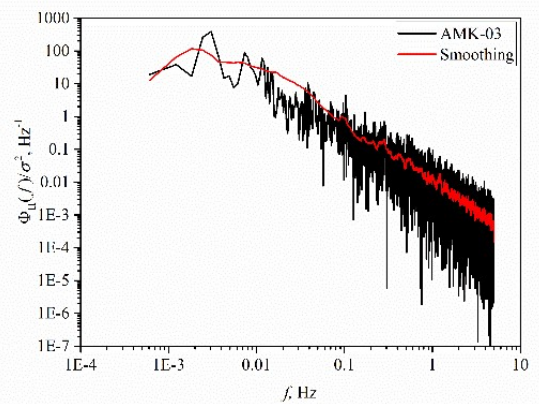

(c)

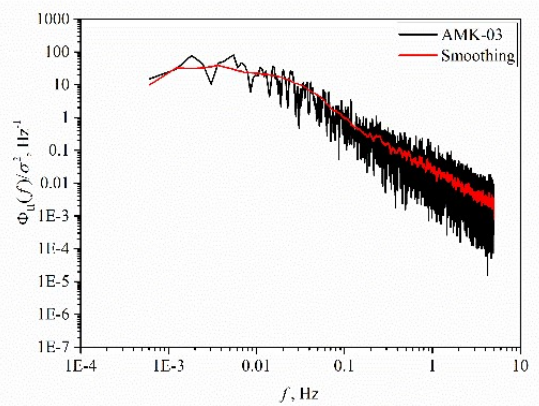

(e)

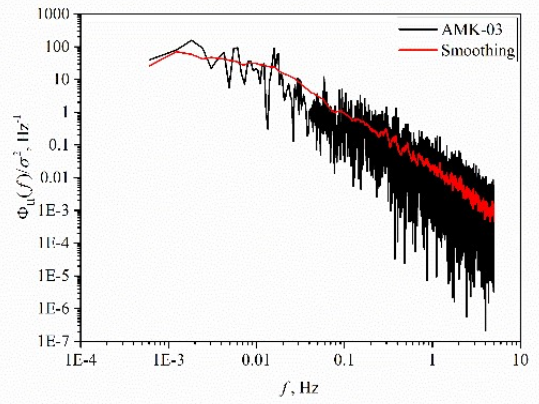

(g)

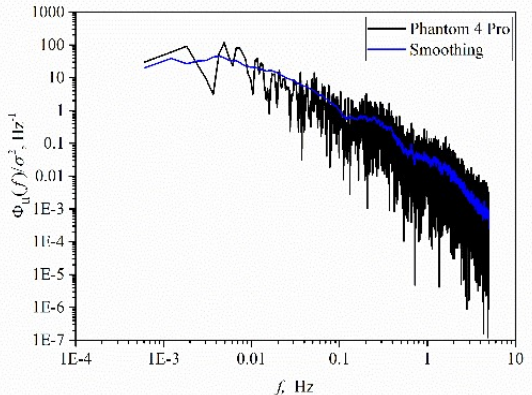

(b)

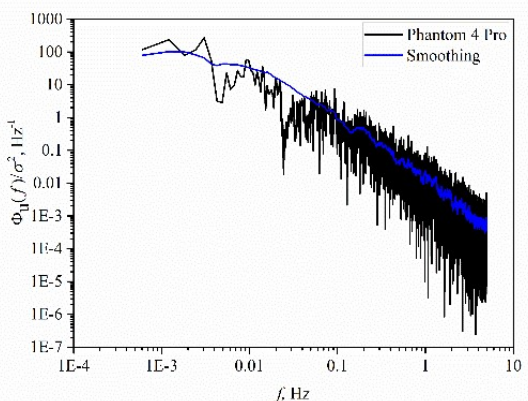

(d)

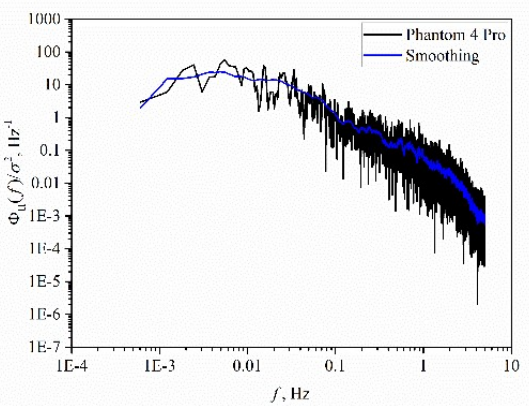

(f)

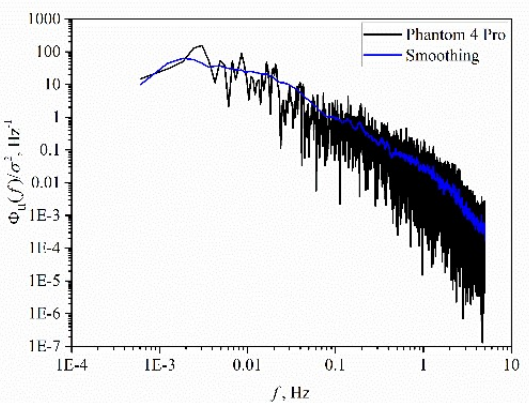

(h)

Figure 7. Longitudinal spectra of turbulence $\Phi_{\mathrm{u}}(f)$ : measured turbulence spectra (black curves), smoothed turbulence spectra obtained from AMK-03 (red curves) and DJI Phantom 4 Pro data (blue curves); $\sigma^{2}$ is the normalization coefficient; (a, b) February 4, (c, d) March 17, (e, f) August 13, and (g, h) November, 15, 2020. 


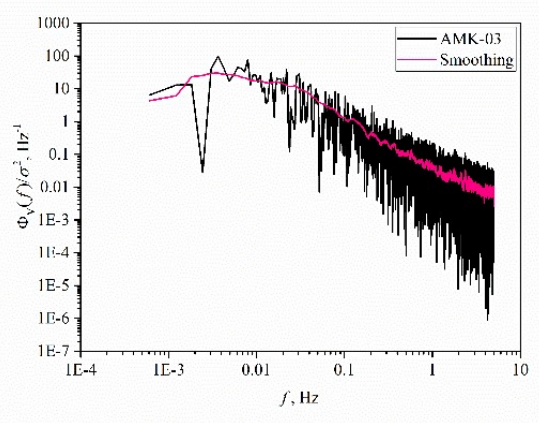

(a)

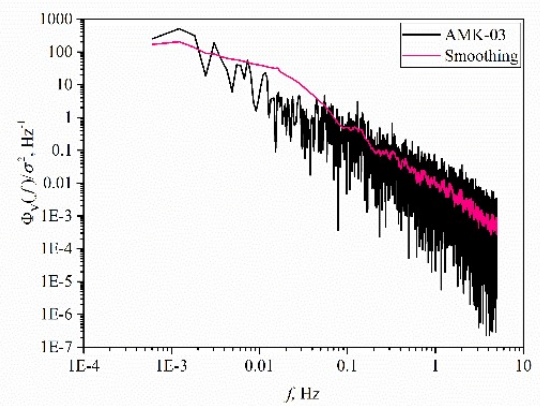

(c)

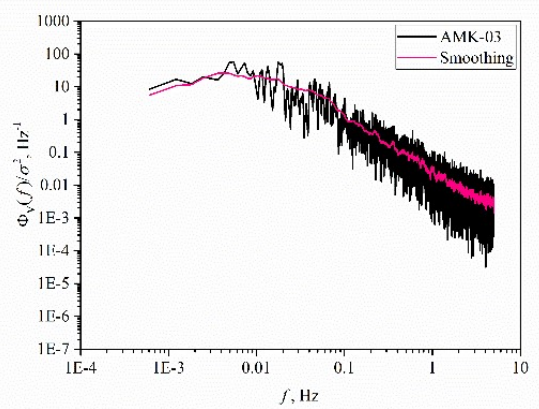

(e)

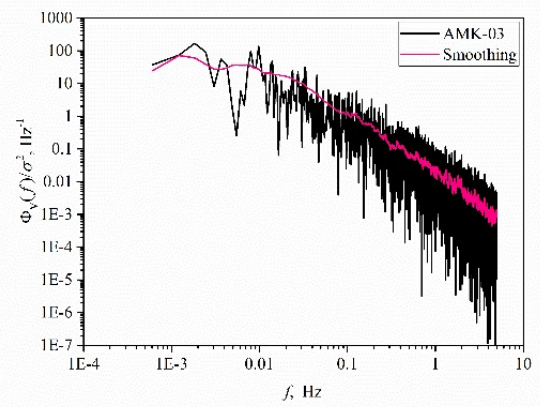

(g)

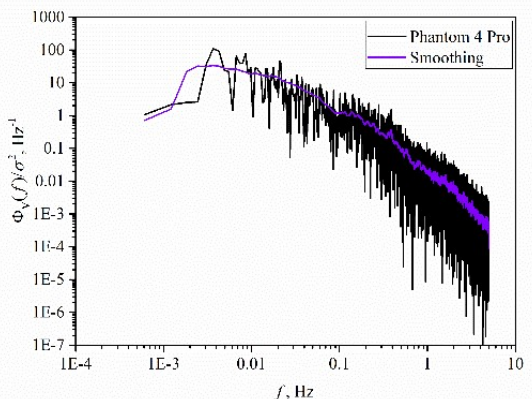

(b)

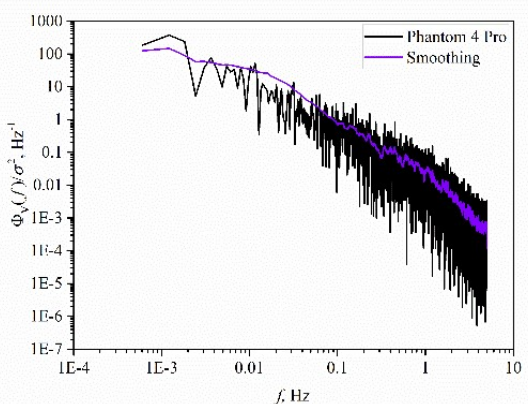

(d)

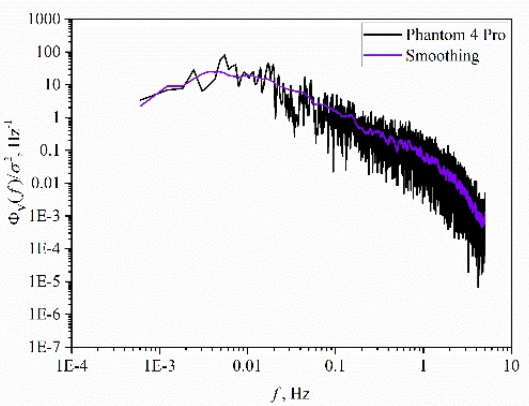

(f)

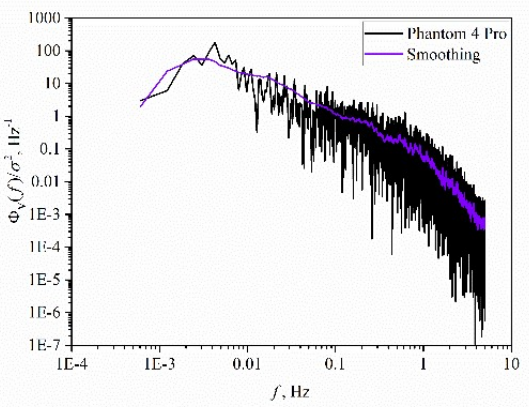

(h)

Figure 8. Lateral spectra of turbulence $\Phi_{\mathrm{v}}(f)$ : measured turbulence spectra (black curves), smoothed turbulence spectra obtained from AMK-03 (pink curves) and DJI Phantom 4 Pro data (purple curves); $\sigma^{2}$ is the normalization coefficient; (a, b) February 4, (c, d) March 17, (e, f) August 13, and (g, h) November, 15, 2020. 


\section{Spectra of turbulence}

Figures 7 and 8 show the results of measurements of the longitudinal and lateral relative turbulence spectra $\Phi_{\mathrm{u}}(f)$ and $\Phi_{\mathrm{v}}(f)$. The turbulence spectra obtained from the data of the AMK-03 ultrasonic weather station and the DJI Phantom 4 Pro quadcopter are shown by the black curves, $\sigma^{2}$ is the normalization coefficient. It can be seen that the values of spectra $\Phi_{\mathrm{u}}(f)$ and $\Phi_{\mathrm{v}}(f)$ vary significantly at minor variations of the frequency $f$. These variations are random oscillations about the main regularities of the turbulence spectra. To reveal these regularities in the turbulence spectra, a smoothing procedure was used.

The result of applying the smoothing procedure is shown in Figs. 7 and 8 by continuous colored curves: red and blue curves for the turbulence spectra of the longitudinal velocity component $\Phi_{\mathrm{u}}(f)$, pink and purple curves for the turbulence spectra of the lateral velocity component $\Phi_{\mathrm{v}}(f)$. Figures $7 \mathrm{a}, \mathrm{c}, \mathrm{e}, \mathrm{g}$ and $8 \mathrm{a}, \mathrm{c}, \mathrm{e}, \mathrm{g}$ depict the results of application of the smoothing procedure of the relative turbulence spectra obtained from the data of the AMK-03 ultrasonic weather station, while Figs. $7 \mathrm{~b}, \mathrm{~d}, \mathrm{f}, \mathrm{h}$ and $8 \mathrm{~b}, \mathrm{~d}, \mathrm{f}, \mathrm{h}$ show those for the DJI Phantom 4 Pro quadcopter data. The figures correspond to the following dates: (a, b) February 4, (c, d) March 17, (e, f) August 13, and (g, h) November, 15, 2020.

Figure 9 compares the smoothed turbulence spectra obtained from the AMK-03 and DJI Phantom 4 Pro data. Similarly to Figs. 7 and 8, red and blue curves for the turbulence spectra of the longitudinal velocity component $\Phi_{\mathrm{u}}(f)$, pink and purple curves for the turbulence spectra of the lateral velocity component $\Phi_{\mathrm{v}}(f)$. It can be seen from Fig. 9 that the turbulence spectra obtained from the AMK-03 and DJI Phantom 4 Pro data generally coincide, and slight differences are observed in the high-frequency range of the spectrum.

It is well known $[48,50]$ that the turbulence spectrum has three main spectral ranges: energy production range, inertial range, and dissipation range. In the energy production range, which contains the main part of the turbulent energy, the energy is generated by buoyancy and shear. In the inertial range, the energy is neither generated nor dissipated, but transferred from large scales to smaller ones. In the dissipation range, the kinetic energy is converted into the internal energy. Consider the behavior of the turbulence spectra in the inertial and energy production ranges.

\section{Inertial and energy production ranges}

In the inertial range, the turbulence spectrum obeys the Kolmogorov-Obukhov "5/3" law [58-50], which was found from dimensional considerations. The von Karman model is generalization of the " $5 / 3$ " law to the frequencies of the energy production range. Therefore, the Kolmogorov-Obukhov law can be found from model (19)-(21) at $\mathrm{L}_{\mathrm{u}} f /\langle\mathrm{u}\rangle \gg 1, \mathrm{~L}_{\mathrm{v}} f /\langle\mathrm{u}\rangle \gg 1$ and $\mathrm{L}_{\mathrm{w}} f /\langle\mathrm{u}\rangle \gg 1$. In the inertial range, the spectral curve has the form

$$
\begin{aligned}
& \frac{\Phi_{\mathrm{u}}(f)}{\sigma_{\mathrm{u}}^{2}} \sim f^{-5 / 3}, \\
& \frac{\Phi_{\mathrm{v}}(f)}{\sigma_{\mathrm{v}}^{2}} \sim f^{-5 / 3}, \\
& \frac{\Phi_{\mathrm{w}}(f)}{\sigma_{\mathrm{w}}^{2}} \sim f^{-5 / 3} .
\end{aligned}
$$

In Fig. 9, the dashed curves show the turbulence spectra corresponding to the Kolmogorov-Obukhov " $5 / 3$ " law, which, as was already noted, keeps true for a homogeneous surface. It was already mentioned that the IMCES SB RAS territory is not homogeneous and has complex orography. Despite the complex orography, the turbulence spectrum in the inertial range obeys the $f^{-5 / 3}$ law, as is clearly seen from Fig. 9. Insignificant 
discrepancies are explained by additional vortices arising as a result of the turbulent flow around the IMCES SB RAS buildings or near the small grove where the $30-\mathrm{m}$ weather tower is installed.

The main characteristics of the energy production range include the longitudinal and lateral scales of turbulence $\mathrm{L}_{\mathrm{u}}$ and $\mathrm{L}_{\mathrm{v}}$, respectively The information about these scales is contained in relative turbulence spectra. In the case of an isotropic atmosphere, the equation for the scale ratio has the form

$$
\frac{\mathrm{L}_{\mathrm{v}}}{\mathrm{L}_{\mathrm{u}}}=0.5 \text {. }
$$

To estimate the scales $\mathrm{L}_{\mathrm{u}}$ and $\mathrm{L}_{\mathrm{v}}$, the von Karman model is used in the approximation of experimental data. The values of the turbulence scales are determined by the equations relating the turbulence scales and the values of the maxima of the functions $f \Phi_{\mathrm{u}}(f)$ and $f \Phi_{\mathrm{v}}(f)$ [51]. In this study, we used the least square fit method for approximation of the experimental data with the von Karman model as the regression curve. In contrast to [51], the maximum was not sought for, and the scales $L_{u}$ and $L_{v}$ were determined directly as the parameters of the best fit when applying the least square fit method. The calculation procedure used in this study is equivalent to the approach outlined in [51].

The values of the turbulence scales are given in Table 3. One can see that the turbulence scales calculated from the AMK-03 and DJI Phantom 4 Pro data coincide to a good accuracy. Keeping in mind that the IMCES SB RAS territory is not homogeneous and has complex orography, we can conclude that the scale ratio is also true to a good accuracy. In Fig. 9, the colored dashed curves are for the best fit curves, red and blue curves are for the turbulence spectra of the longitudinal component $\Phi_{\mathrm{u}}(f)$, and pink and purple curves are for the turbulence spectra of the lateral component $\Phi_{\mathrm{v}}(f)$. Figure 9 demonstrates the good agreement for the best fit curves for the turbulence spectra obtained from the ANK-03 and DJI Phantom 4 Pro data. 


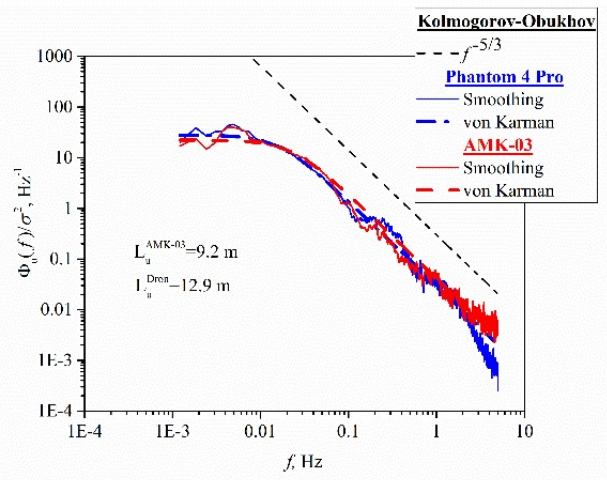

(a)

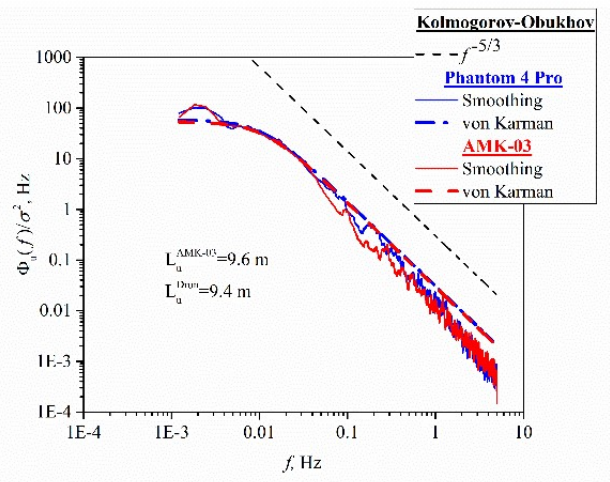

(c)

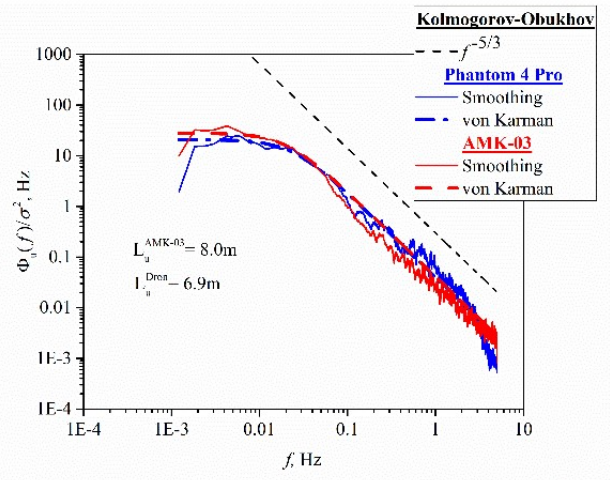

(e)

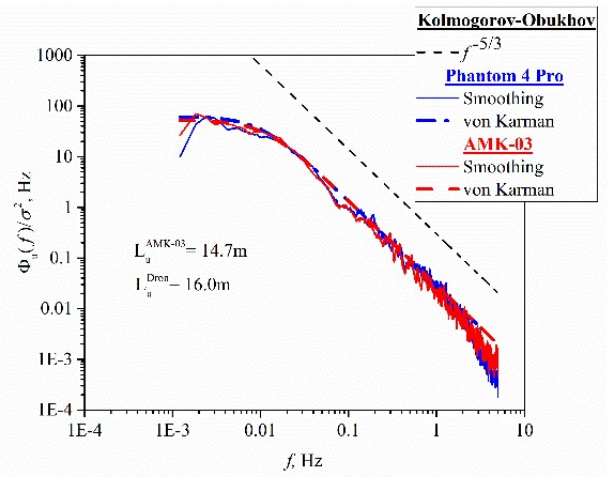

(g)

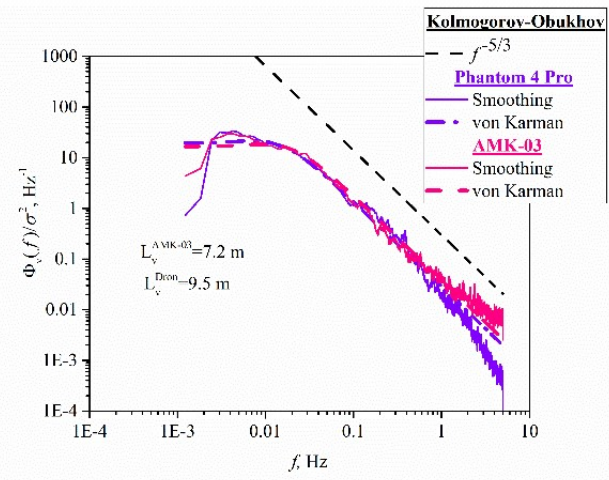

(b)

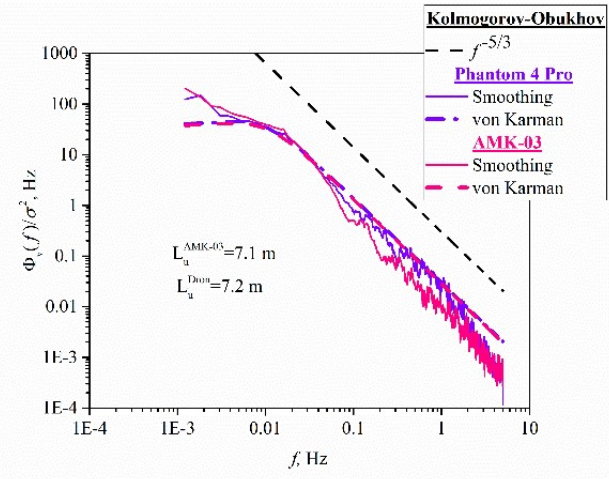

(d)

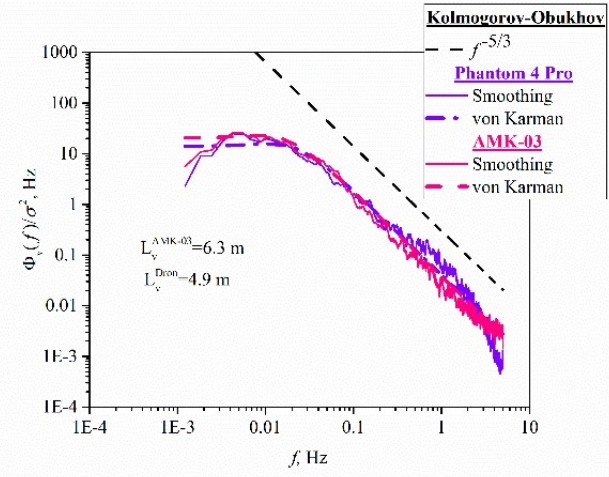

(f)

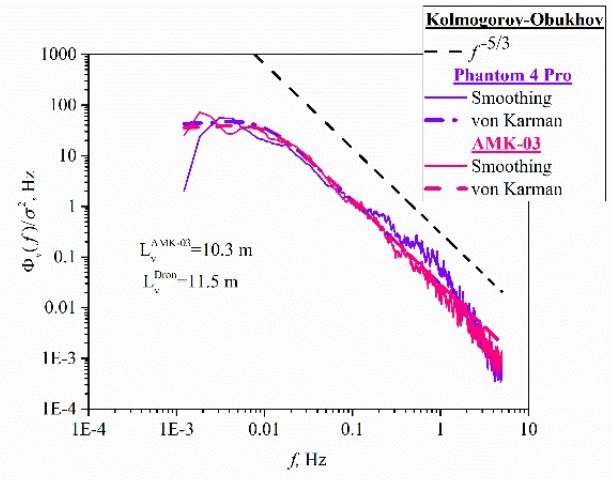

(h)

Figure 9. Longitudinal $\Phi_{\mathrm{u}}(f)$ and lateral $\Phi_{\mathrm{v}}(f)$ turbulence spectra upon application of the smoothing procedure: (red and blue curves) smoothed longitudinal turbulence spectra obtained from the AMK-03 and DJI Phantom 4 Pro data, re- 
spectively, (pink and purple curves) smoothed turbulence spectra obtained from the AMK-03 and DJI Phantom 4 Pro data; $\sigma^{2}$ is the normalization coefficient; (a, b) February 4, (c, d) March 17, (e, f) August 13, and (g, h) November, 15, 2020.

Table 3. Integral scales of turbulence.

\begin{tabular}{cccc}
\hline & $\mathrm{L}_{\mathrm{u}}$ & $\mathrm{L}_{\mathrm{v}}$ & $\mathrm{L}_{\mathrm{v}} / \mathrm{L}_{\mathrm{u}}$ \\
\hline AMK-03 & & February 4,2020 & \\
\hline DJI Phantom 4 Pro & 9.2 & 7.2 & 0.78 \\
\hline AMK-03 & 12.9 & 9.5 & 0.74 \\
\hline DJI Phantom 4 Pro & 9.6 & March 17, 2020 & 0.74 \\
\hline & 9.4 & 7.1 & 0.77 \\
\hline AMK-03 & & August 13, 2020 & 0.79 \\
DJI Phantom 4 Pro & 8.0 & 6.3 & 0.71 \\
\hline & 6.9 & 4.9 & 0.70 \\
\hline AMK-03 & & November 15, 2020 & 0.72 \\
\hline DJI Phantom 4 Pro & 14.7 & 10.3 & 11.5 \\
\hline
\end{tabular}

\section{Discussion}

The studies of the use of DJI Phantom 4 Pro in the hover mode in combination with the AMK-03 ultrasonic weather stations show that the quadcopter allows obtaining turbulence spectra with high spatial resolution in the atmosphere in areas with complex orography in hard-to-reach places, under various weather conditions, as well as in different seasons: winter, spring, summer and fall.

The measured values of the longitudinal and lateral spectra vary significantly with insignificant variations of the frequency. Therefore, we used the smoothing procedure to study main regularities in the behavior of turbulence in the inertial and energy production ranges. To estimate the longitudinal and lateral scales of turbulence, the least square fit method was used with the von Karman model as a regression curve.

The longitudinal and lateral turbulence spectra obtained with the DJI Phantom 4 Pro and AMK-03 are generally the same with minor differences observed in the highfrequency range of the spectrum. Discrepancies in the high-frequency spectral range are also observed in the behavior of the time series of the longitudinal and lateral components of wind velocity measured by different methods. The behavior of the turbulence spectra in the inertial range shows that they obey the Kolmogorov-Obukhov "5/3" law. In the energy production range, the longitudinal and lateral turbulence scales and their ratio measured by both the DJI Phantom 4 Pro and AMK-03 coincide to a good accuracy.

Discrepancies in the behavior of the turbulence spectra obtained experimentally by different methods can be explained as follows. First, for safety reasons, the quadcopter was at a distance of $\sim 10 \mathrm{~m}$ from AMK-03 during the experiment. Measurements of the integral turbulence scales show that they are of the same order of magnitude as the distance from AMK-03 to DJI Phantom 4 Pro. This relation between the scales and the distances means that the wind velocity fields at the AMK-03 and quadcopter locations are partially correlated. Therefore, it makes no sense to talk about complete equality of the measured data and, consequently, the turbulence spectra should differ.

Second, experimental studies were carried out at the territory with complex orography. The presence of the park zone, institute buildings, and highways means that the territory is inhomogeneous, which leads to significant deviations from homogeneity and isotropy. As a result, the behavior of the wind velocity field and its characteristics is different for the AMK-03 and quadcopter locations. This difference is most pronounced in the behavior of the ratio of turbulence scales. For the homogeneous and isotropic atmos- 
phere this ratio is $\mathrm{L}_{\mathrm{v}} / \mathrm{L}_{\mathrm{u}}=0.5$, but in our experiment, as can be seen from Table 3, $\mathrm{L}_{\mathrm{v}} / \mathrm{L}_{\mathrm{u}} \sim 0.8$ for AMK-03 and $\mathrm{L}_{\mathrm{v}} / \mathrm{L}_{\mathrm{u}} \sim 0.7$ for DJI Phantom 4 Pro on August 13 .

It was shown in [47] that the results of investigation of the turbulence spectra in the inertial and energy production ranges with the quadcopter in the altitude holding mode over a homogeneous territory are in a good agreement with the theory of homogeneous and isotropic turbulence and with the data of measurement by acoustic anemometry. This study allows us to conclude that in these ranges at the territory with complex orography, the behavior of the turbulence spectrum measured by the quadcopter agrees with objective data on the state of atmospheric turbulence. Thus, the results obtained allow us to assert that a rotary-wing UAV can serve as a tool having the great potential for diagnostics of the atmospheric boundary layer. Due to the capability of providing data on the state of atmospheric turbulence with high spatial resolution, the quadcopter is a promising tool for solving problems of controlling the UAV movement under bad weather conditions, as well as problems of wind energy, climatic measurements in an urban environment, etc.

From the scientific and practical points of view, it is of great interest to monitor the state of atmospheric turbulence at various spatial points of the studied area. From this point of view, further work on the use of a quadcopter for low-altitude sensing is associated with the use of a quadcopter swarm to determine profiles of atmospheric turbulence in both the vertical and horizontal planes.

Author Contributions: Conceptualization, AS; methodology, AS; software, AA, ES; validation, AK, AT and AM; investigation, AS, AA; data curation, AK, AT, AM; writing-original draft preparation, OP; writing - review and editing, AS. supervision, AS; project administration, AS.

Funding: P The study was supported by the Russian Foundation for Basic Research (project no. 19-29-06066 mk).

Conflicts of Interest: The authors declare no conflict of interest. The funders had no role in the design of the study; in the collection, analyses, or interpretation of data; in the writing of the manuscript, or in the decision to publish the results.

\section{References}

1. Cornman, L.B.; Chan, W.N. Summary of a Workshop on Integrating Weather into Unmanned Aerial System Traffic Management. Bulletin of the American Meteorological Society 2017, 98(10), ES257-ES259.

2. Shakhatreh, H.; Sawalmeh, A.; Al-Fuqaha, A.; Dou, Z.; Almaita, E.; Khalil, I.; Othman, N.; Khreishah, A.; Guizani, M. Unmanned Aerial Vehicles (UAVs): A Survey on Civil Applications and Key Research Challenges. IEEE Access 2019, 7, 4857248634.

3. Hatfield, M.; Cahill, C.; Webley, P.; Garron, J.; Beltran, R. Integration of Unmanned Aircraft Systems into the National Airspace System-Efforts by the University of Alaska to Support the FAA/NASA UAS Traffic Management Program. Remote Sensing 2020, 12, 3112.

4. Kazarin, P.; Golubev, V.; MacKunis, W.; Moreno, C. Robust Nonlinear Tracking Control for Unmanned Aircraft in the Presence of Wake Vortex. Electronics 2021, 10, 1890.

5. Borener, S.; Trajkov, S.; Balakrishna, P. Design and development of an integrated safety assessment model for NextGen. In Proceedings of the International Annual Conference of the American Society for Engineering Management, San Antonio, TX, USA, 10-13 June 2012.

6. Abichandani, P.; Lobo, D.; Ford, G.; Bucci D.; Kam, M. Wind Measurement and Simulation Techniques in Multi-Rotor Small Unmanned Aerial Vehicles," IEEE Access, 2020 8, 54910-54927.

7. Stith, J.L.; Baumgardner, D.; Haggerty, J.; Hardesty, M.; Lee, W.; Lenschow, D.; Pilewskie, P.; Smith, P.L.; Steiner, M.; Vömel, H. 100 Years of Progress in Atmospheric Observing Systems Meteorological Monographs 2018, 59.

8. Frehlich, R.; Meillier, Y.; Jensen, M.A.; Balsley, B.B. Turbulence measurements with the CIRES tethered lifting system during CASES-99: Calibration and spectral analysis of temperature and velocity. Journal of the Atmospheric Sciences 2003, 60, 24872495.

9. Yang, S.; Petersen, G.N.; von Löwis, S.; Preißler, J.; Finger, D. Determination of eddy dissipation rate by Doppler lidar in Reykjavik, Iceland. Meteorological Applications 2020, 27, e1951. 
10. Hocking, W.K.; Röttger, J.; Palmer, R.D.; Sato, T.; Chilson, P.B. Atmospheric Radar, Cambridge University Press, Cambridge, $\mathrm{UK}, 2016$.

11. Lundquist, J.K.; Wilczak, J.M.; Ashton, R.; Bianco, L.; Brewer, W.A.; Choukulkar, A.; Clifton, A.; Debnath, M.; Delgado, R.; Friedrich, K.; Gunter, S.; Hamidi, A.; Iungo, G. V.; Kaushik, A.; Kosović, B.; Langan, P.; Lass, A.; Lavin, E.; Lee, J. C.; McCaffrey, K. L.; Newsom, R. K.; Noone, D. C.; Oncley, S. P.; Quelet, P. T.; Sandberg, S. P.; Schroeder, J. L.; Shaw, W. J.; Sparling, L.; Martin, C. S.; Pe, A. S.; Strobach, E.; Tay, K.; Vanderwende, B. J.; Weickmann, A.; Wolfe, D.; Worsnop, R. Assessing State-ofthe-Art Capabilities for Probing the Atmospheric Boundary Layer: The XPIA Field Campaign. Bulletin of the American Meteorological Society 2017, 98(2), 289-314.

12. Kral, S.T.; Reuder, J.; Vihma, T.; Suomi, I.; O’Connor, E.; Kouznetsov, R.; Wrenger, B.; Rautenberg, A.; Urbancic, G.; Jonassen, M.O.; Båserud, L.; Maronga, B.; Mayer, S.; Lorenz, T.; Holtslag, A.A.M.; Steeneveld, G.-J.; Seidl, A.; Müller, M.; Lindenberg, C.; Langohr, C.; Voss, H.; Bange, J.; Hundhausen, M.; Hilsheimer, P.; Schygulla, M. Innovative Strategies for Observations in the Arctic Atmospheric Boundary Layer (ISOBAR) - The Hailuoto 2017 Campaign. Atmosphere 2018, 9, 268.

13. Leosphere, "Windcube," Vaisala, [Online]. Available: https:/www.vaisala.com/en/wind-lidars/wind-energy/windcube/.

14. METEK Meteorologische Messtechnik GmbH, [Online]. Available: https://metek.de/product-group/doppler-sodar/.

15. Scintec, [Online]. Available: https://www.scintec.com/.

16. Azbukin, A.A.; Bogushevich, A.Ya.; Korolkov, V.A.; Tikhomirov, A.A.; Shelevoi, V.D. A field version of the AMK-03 automated ultrasonic meteorological complex Russian Meteorology and Hydrology 2009, 34(2), 133-136.

17. Azbukin, A.A.; Bogushevich, A.Ya.; Kobzev, A.A.; et al. AMK-03 Automatic weather stations, their modifications and applications Sensors and systems 2012, 3(154), 47-52.

18. Palomaki, R.T.; Rose, N.T.; van den Bossche, M.; Sherman, T.J.; De Wekker, S.F.J. Wind estimation in the lower atmosphere using multirotor aircraft. Journal of Atmospheric and Oceanic Technology 2017, 34, 1183-1190.

19. González-Rocha, J.; De Wekker, S.F.J.; Ross, S.D.; Woolsey, C.A. Wind profiling in the lower atmosphere from wind-induced perturbations to multirotor. UAS Sensors 2020, 20, 1341.

20. González-Rocha, J.; Woolsey, C. A., Cornel Sultan measuring atmospheric winds from quadrotor motion AIAA Atmospheric Flight Mechanics Conference, Grapevine, Texas, USA, 2017.

21. González-Rocha, J.; Woolsey, C.A.; Cornel Sultan, C.; De Wekker S.F.J. Sensing Wind from Quadrotor Motion. Journal of Guidance, Control, and Dynamics 2019, 42, 1-18.

22. Reuder, J.; Brisset, P.; Jonassen, M.; Muller, M.; Mayer, S. The Small Unmanned Meteorological Observer SUMO: A new tool for atmospheric boundary layer research. Meteorologische Zeitschrift 2009, 18(2), 141-147.

23. Varentsov, M.; Stepanenko, V.; Repina, I.; Artamonov, A.; Bogomolov, V.; Kuksova, N.; Marchuk, E.; Pashkin, A.; Varentsov, A. Balloons and Quadcopters: Intercomparison of Two Low-Cost Wind Profiling Methods. Atmosphere 2021, $12,380$.

24. Rautenberg, A.; Graf, M.; Wildmann, N.; Platis, A.; Bange, J. Reviewing Wind Measurement Approaches for Fixed-Wing Unmanned Aircraft. Atmosphere 2018, 9, 422.

25. Shimura, T.; Inoue, M.; Tsujimoto, H.; Sasaki, K.; Iguchi, M. Estimation of Wind Vector Profile Using a Hexarotor Unmanned Aerial Vehicle and Its Application to Meteorological Observation up to $1000 \mathrm{~m}$ above Surface. Journal of Atmospheric and Oceanic Technology 2018, 35, 1621-1631.

26. Neumann, P.P.; Bartholmai, M. Real-time wind estimation on a micro unmanned aerial vehicle using its internal measurement unit. Sens. Actuators 2015, 235A, 300-310.

27. Allison, S.; Bai, H.; Jayaraman, B. Wind estimation using quadcopter motion: A machine learning approach. Aerospace Science and Technology 2020, 98, 105699.

28. Wang, L.; Misra, G.; Bai, X.; A K Nearest Neighborhood-Based Wind Estimation for Rotary-Wing VTOL UAVs. Drones 2019, $3(2), 31$.

29. Chechin, D.G.; Artamonov, A.Y.; Bodunkov, N.E.; Zhivoglotov, D.N.; Zaytseva, D.V.; Kalyagin, M.Y.; Kouznetsov, D.D.; Kounashouk, A.A.; Shevchenko, A.M.; Shestakova, A.A. Experience of Studying the Turbulent Structure of the Atmospheric Boundary Layer Using an Unmanned Aerial Vehicle. Izvestiya, Atmospheric and Oceanic Physics 2021, 57(5), 526-532.

30. Simma, M.; Mjøen, H.; Boström T. Measuring Wind Speed Using the Internal Stabilization System of a Quadrotor Drone. Drones, 2020, 4, 23.

31. Wang, J.Y.; Luo, B.; Zeng, M.; Meng, Q. A Wind Estimation Method with an Unmanned Rotorcraft for Environmental Monitoring Tasks. Sensors 2018, 18(12), 4504

32. Brossard, M.; Condomines, J.-P.; Bonnabel, S. "Tightly Coupled Navigation and Wind Estimation for Mini UAVs," AIAA 20181843. 2018 AIAA Guidance, Navigation, and Control Conference. January 2018.

33. Wang, B.H.; Wang, D.B.; Ali, Z.A.; Ting, B.T.; Wang, H. An overview of various kinds of wind effects on unmanned aerial vehicle. Measurement and Control 2019, 52(7-8), 731-739. 
34. Al-Ghussain, L.; Bailey, S. C. C. An approach to minimize aircraft motion bias in multi-hole probe wind measurements made by small unmanned aerial systems. Atmospheric Measurement Techniques 2021 14(1), $173-184$.

35. Sekula, P.; Zimnoch, M.; Bartyzel, J.; Kud, M.; Necki, J.; Bokwa, A. Ultra-Light Airborne Measurement System for Investigation of Urban Boundary Layer Dynamics. Sensors 2021, 21, 2920.

36. Reuder, J.; Jonassen, M.O. First results of turbulence measurements in a wind park with the Small Unmanned Meteorological Observer SUMO Energy Procedia 2012, 24, 176-185.

37. Reuder, J.; Jonassen, M.O. Proof of concept for wind turbine wake investigations with the RPAS SUMO Energy Procedia 2016, $94,452-461$.

38. Reineman, B.D. Development and Testing of Instrumentation for UAV-Based Flux Measurements within Terrestrial and Marine Atmospheric Boundary Layers. Journal of Atmospheric and Oceanic Technology 2013, 30, 1295-1319.

39. Reineman, B.D.; Lenain, L.; Melville, W.K. The Use of Ship-Launched Fixed-Wing UAVs for Measuring the Marine Atmospheric Boundary Layer and Ocean Surface Processes. Journal of Atmospheric and Oceanic Technology 2016, 33, $2029-2052$.

40. Balsley, B.B.; Lawrence, D.A.; Fritts, D.C.; Wang, L.; Wan, K.; Werne, J. Fine Structure, Instabilities, and Turbulence in the Lower Atmosphere: High-Resolution In Situ Slant-Path Measurements with the DataHawk UAV and Comparisons with Numerical Modeling. Journal of Atmospheric and Oceanic Technology 2018, 35, 619-642.

41. Rautenberg, A.; Allgeier, J.; Jung, S.; Bange, J. Calibration Procedure and Accuracy of Wind and Turbulence Measurements with Five-Hole Probes on Fixed-Wing Unmanned Aircraft in the Atmospheric Boundary Layer and Wind Turbine Wakes. Atmosphere 2019, 10, 124.

42. Fuertes, F.C.; Wilhelm, L.; Porté-Agel, F. Multirotor UAV-Based Platform for the Measurement of Atmospheric Turbulence: Validation and Signature Detection of Tip Vortices of Wind Turbine Blades. J. Atmos. Oceanic Technol. 2019, 36(6), 941-955.

43. Kim M.; Kwon, B.H. Estimation of Sensible Heat Flux and Atmospheric Boundary Layer Height Using an Unmanned Aerial Vehicle. Atmosphere 2019, 10, 363.

44. Luce, H.; Kantha, L.; Hashiguchi, H.; Lawrence, D. Estimation of Turbulence Parameters in the Lower Troposphere from ShUREX (2016-2017) UAV Data. Atmosphere 2019, 10, 384.

45. Witte, B.M.; Singler, R.F.; Bailey, S.C.C. Development of an Unmanned Aerial Vehicle for the Measurement of Turbulence in the Atmospheric Boundary Layer. Atmosphere 2017, 8, 195.

46. Wildmann, N.; Eckert, R.; Dörnbrack, A.; Gisinger, S.; Rapp, M.; Ohlmann, K.; van Niekerk, A. (2021). In Situ Measurements of Wind and Turbulence by a Motor Glider in the Andes. Journal of Atmospheric and Oceanic Technology 2021, 38(4), 921-935.

47. Shelekhov, A.; Afanasiev, A.; Shelekhova, E.; Kobzev, A.; Tel'minov, A.; Molchunov, A.; \& Poplevina, O. Using Small Unmanned Aerial Vehicles for Turbulence Measurements in the Atmosphere. Izvestiya, Atmospheric and Oceanic Physics 2021, 57, 533-545.

48. Monin, A. S.; Yaglom, A. M. Statistical Hydromechanics. Part 2. Turbulent Mechanics, Nauka, Moscow, 1967.

49. Stull, R.B. An Introduction to Boundary Layer Meteorology, Kluwer Academic Publishers, Netherlands 1989.

50. Kaimal, J.C.; Finnigan, J.J. Atmospheric Boundary Layer Flows. Their Structure and Measurement, Oxford University Press, New York Oxford, 1994.

51. Teunissen, H. W. Characteristics of the Mean Wind and Turbulence in the Planetary Boundary Layer. UTIAS Review 1970, 32.

52. Tieleman, H. W. Universality of velocity spectra. Journal of Wind Engineering and Industrial Aerodynamics 1995, 56(1), 55-69.

53. Mahony, R.; Kumar, V.; Corke, P. Multirotor aerial vehicles: Modeling, estimation, and control of quadrotor. IEEE Robotics Automation Magazine 2012, 19, 20-32. 